\title{
TWISTED WHITEHEAD PRODUCTS
}

\author{
HOWARD J. MARCUM
}

\begin{abstract}
By combining the Hopf construction and the Whitehead product map construction we introduce twisted Whitehead products and then extend several well-known properties of Whitehead products to the twisted situation.
\end{abstract}

1. Introduction. Universally defined homotopy pairings $\pi_{1}^{A}(X) \times \pi_{1}^{B}(X) \rightarrow$ $\pi_{1}^{Y}(X)$ are classified by the elements of the group $\pi_{1}^{Y}(\Sigma A \vee \Sigma B)$. Those elements in the image of the boundary homomorphism

$$
\pi_{2}^{Y}(\Sigma A \times \Sigma B, \Sigma A \vee \Sigma B) \rightarrow \pi_{1}^{Y}(\Sigma A \vee \Sigma B)
$$

correspond to the $\gamma^{\dagger}$-homotopy operations of Hilton [4, p. 189] and have the property that they vanish under suspension. For $Y=A \wedge B$, the generalized Whitehead product $[\alpha, \beta] \in \pi_{1}^{A \wedge B}(X)$ of $\alpha \in \pi_{1}^{A}(X)$ and $\beta \in \pi_{1}^{B}(X)$ is an example of such an operation.

Alternatively the $\gamma^{\dagger}$-homotopy operations can be characterized as those classes $\gamma: \Sigma Y \rightarrow \Sigma A \vee \Sigma B$ which are trivial on both $\Sigma A$ and $\Sigma B$. Recent work of H. J. Baues [3, p. 171] focuses attention as well on classes $\gamma$ : $\Sigma Y \rightarrow \Sigma A \vee \Sigma B$ which are trivial on $\Sigma A$ but not necessarily on $\Sigma B$. For $Y=A \wedge B$ we shall provide here examples of these latter operations by introducing twisted Whitehead products. Our purpose is to generalize some well-known properties of the usual Whitehead product to the twisted case. For properties of the usual Whitehead product the reader is referred to the classic article of Arkowitz [1].

2. Notation. With one exception we work entirely within the based topological category. Furthermore we assume that all spaces are well pointed; i.e., the inclusion of the base point is an (unbased) cofibration. All homotopies preserve base points.

$A * B$ denotes the unreduced topological join of $A$ and $B$ (this is the one exception above) and is provided with the variable base point as explained in [5]. We recall that since all spaces are well pointed, the composite

$$
\nu: A * B \stackrel{\kappa}{\rightarrow} \Sigma(A \times B) \stackrel{\Sigma q}{\rightarrow} \Sigma(A \wedge B)
$$

is a (based) homotopy equivalence. (Here $\kappa$ collapses the "ends" of the join

Presented to the Society, August 17, 1977; received by the editors February 9, 1978.

AMS (MOS) subject classifications (1970). Primary 55E15, 55E35.

Key words and phrases. Whitehead product, Hopf construction, clutching function, fiberwise join. 
and $q: A \times B \rightarrow A \wedge B$ is the usual quotient.) We use $\nu$ to regard $A * B$ as an $H$-cogroup.

Given a map $f: A \times B \rightarrow C$ the Hopf construction on $f$ is the map $h(f)$ : $A * B \rightarrow \Sigma C$ given by

$$
h(f)[a, b, t]=[f(a, b), t], \quad a \in A, b \in B, 0 \leqslant t \leqslant 1 .
$$

By means of $\nu, h(f)$ may be regarded as a class $h(f): \Sigma(A \wedge B) \rightarrow \Sigma C$. Suppose that $f$ has type $(e, d)$. That is, $\left.f\right|_{A \times\{*\}} \simeq e: A \rightarrow C$ and $\left.f\right|_{(*) \times B} \simeq d$ : $B \rightarrow C$. Then one easily verifies:

(2.1) Regarding $h(f) \in \pi_{1}^{A \wedge B}(\Sigma C)$ we have

$$
h(f) \Sigma q \simeq-\Sigma e \Sigma \mathrm{pr}_{A}+\left(\Sigma f-\Sigma d \Sigma \mathrm{pr}_{B}\right)
$$

where $\operatorname{pr}_{A}$ and $\mathrm{pr}_{B}$ are the two projection maps on $A \times B$.

(2.2) We shall also need the action of $\pi_{1}^{A}(Z)$ on $\pi_{1}^{A \wedge B}(Z)$ which is defined as follows. Given $\alpha \in \pi_{1}^{A}(Z)$ and $\xi \in \pi_{1}^{A \wedge B}(Z), \xi^{\alpha} \in \pi_{1}^{A \wedge B}(Z)$ is defined by

$$
\xi^{\alpha} \Sigma q \simeq-\alpha \Sigma \mathrm{pr}_{A}+\xi \Sigma q+\alpha \Sigma \mathrm{pr}_{A}
$$

3. The twisted Whitehead product map. From [1] we recall that given spaces $A$ and $B$ the (generalized) Whitehead product map $W: A * B \rightarrow \Sigma A \vee \Sigma B$ is defined by the formula

$$
W[a, b, t]= \begin{cases}(*,[b, 1-2 t]), & 0 \leqslant t \leqslant 1 / 2 \\ ([a, 2 t-1], *), & 1 / 2 \leqslant t \leqslant 1\end{cases}
$$

By combining the Whitehead product map construction and the Hopf construction we obtain the twisted Whitehead product map.

(3.1) Definition. For a fixed map $f: A \times B \rightarrow B$ the twisted Whitehead product map $W_{f}: A * B \rightarrow \Sigma A \vee \Sigma B$ is defined by

$$
W_{f}[a, b, t]= \begin{cases}(*,[f(a, b), 2 t]), & 0 \leqslant t \leqslant 1 / 2, \\ ([a, 2-2 t], *), & 1 / 2 \leqslant t \leqslant 1 .\end{cases}
$$

Observe that $W_{f}$ is the composite

$$
\begin{aligned}
A * B & \stackrel{\kappa}{\rightarrow} \Sigma(A \times B) \stackrel{r}{\rightarrow} \Sigma(A \times B) \\
& \stackrel{\omega}{\rightarrow} \Sigma(A \times B) \vee \Sigma(A \times B) \stackrel{\Sigma \mathrm{pr}_{A} \vee-\Sigma f}{\rightarrow} \Sigma A \vee \Sigma B
\end{aligned}
$$

where $r$ denotes reversal of parameter and $\omega$ is the suspension comultiplication. Thus the class of $W_{f}$ depends only on the class of $f$.

(3.2) (i) If $f=\operatorname{pr}_{B}: A \times B \rightarrow B$ then the composite

$$
A * B \stackrel{W_{f}}{\rightarrow} \Sigma A \vee \Sigma B \stackrel{r \vee r}{\rightarrow} \Sigma A \vee \Sigma B
$$

is the generalized Whitehead product map for $A$ and $B$.

(ii) On $\Sigma A, W_{f}$ is trivial and on $\Sigma B, W_{f}$ is the Hopf construction on $f$. That 
is, the diagram

is homotopy commutative.

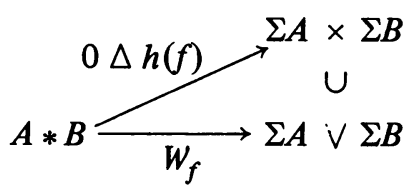

Since $\Sigma(\Sigma A \times \Sigma B)$ homotopy retracts onto $\Sigma(\Sigma A \vee \Sigma B)$, (3.2) (ii) implies:

(3.3) Proposition. The diagram

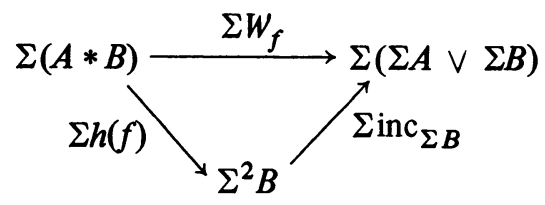

is homotopy commutative.

Observe that since $h\left(\operatorname{pr}_{B}\right) \simeq 0$, (3.3) reduces to $\Sigma W \simeq 0$ in the untwisted case.

(3.4) Definition. For any space $Z$ the twisted Whitehead product pairing

$$
[,]_{f}: \pi_{1}^{A}(Z) \times \pi_{1}^{B}(Z) \rightarrow \pi(A * B, Z)
$$

is defined by $[\alpha, \beta]_{f}=W_{f}(\alpha \nabla \beta)$ for $\alpha \in \pi_{1}^{A}(Z), \beta \in \pi_{1}^{B}(Z)$.

By (3.2)(i) and (3.3), we have

$$
\begin{aligned}
& {[\alpha, \beta]_{\mathrm{pr}_{B}}=[-\alpha,-\beta](=\text { usual Whitehead product }),} \\
& \Sigma[\alpha, \beta]_{f}=\Sigma(\beta h(f)) .
\end{aligned}
$$

4. The commutator definition. The untwisted Whitehead product pairing has an alternative definition in terms of commutators [1, Theorem 2.4]. The twisted analogue is given in the following theorem.

(4.1) Theorem. Suppose $f: A \times B \rightarrow B$ has type $(e, d) ; \alpha \in \pi_{1}^{A}(Z), \beta \in$ $\pi_{1}^{B}(Z)$. Then

$$
[\alpha, \beta]_{f} \equiv\left(\beta \Sigma f-\alpha \Sigma \mathrm{pr}_{A}\right) \kappa \simeq \theta \kappa
$$

where

$$
\theta \equiv\left(\alpha \Sigma \mathrm{pr}_{A}-\beta \Sigma e \Sigma \mathrm{pr}_{A}\right)+\left(\left(\beta \Sigma f-\alpha \Sigma \mathrm{pr}_{A}\right)-\beta \Sigma d \Sigma \mathrm{pr}_{B}\right)
$$

Hence regarding $[\alpha, \beta]_{f} \in \pi_{1}^{A \wedge B}(Z)$ we have

$$
[\alpha, \beta]_{f} \Sigma q \simeq\left(\alpha \Sigma \mathrm{pr}_{A}-\beta \Sigma e \Sigma \mathrm{pr}_{A}\right)+\left(\left(\beta \Sigma f-\alpha \Sigma \mathrm{pr}_{A}\right)-\beta \Sigma d \Sigma \mathrm{pr}_{B}\right) \text {. }
$$

(4.2) LEMMA. The co-operator map

$$
c: \Sigma(A \times B) \rightarrow \Sigma A \vee \Sigma B \vee \Sigma(A \times B)
$$

associated to the cofibration $A \vee B \rightarrow A * B \stackrel{\kappa}{\rightarrow} \Sigma(A \times B)$ is the composite

$$
\begin{aligned}
X \stackrel{\omega}{\rightarrow} X \vee X \stackrel{1 \vee \omega}{\rightarrow} X \vee X \vee X & \stackrel{1 \vee T}{\rightarrow} X \vee X \vee X \\
& -\Sigma \mathrm{pr}_{A} \vee \Sigma \mathrm{pr}_{B} \vee 1 \\
\rightarrow & \Sigma A \vee \Sigma B \vee X
\end{aligned}
$$


where $X=\Sigma(A \times B), \omega=$ suspension comultiplication and $T$ is interchange of factors.

Proof. A routine check.

Proof of (4.1). Let $\psi$ denote the composite

$$
\Sigma A \vee \Sigma B \stackrel{\omega \vee 1}{\rightarrow} \Sigma A \vee \Sigma A \vee \Sigma B \stackrel{-\alpha \nabla \beta \Sigma e \nabla(-\beta \Sigma d)}{\rightarrow} Z
$$

Then a short computation shows that the composite

$$
\Sigma(A \times B) \stackrel{c}{\rightarrow} \Sigma A \vee \Sigma B \vee \Sigma(A \times B) \stackrel{\psi \nabla\left(\beta \Sigma f-\alpha \Sigma \mathrm{pr}_{A}\right)}{\rightarrow} Z
$$

with $c$ as in (4.2) is just $\theta$. But this implies $\theta \kappa \simeq\left(\beta \Sigma f-\alpha \Sigma \operatorname{pr}_{A}\right) \kappa=[\alpha, \beta]_{f}$, as desired.

(4.3) Since $\operatorname{pr}_{B}: A \times B \rightarrow B$ has type (0,1), (4.1) and (3.2)(i) show that the untwisted Whitehead product satisfies

$$
[\alpha, \beta] \Sigma q \simeq-\alpha \Sigma \mathrm{pr}_{A}-\beta \Sigma \mathrm{pr}_{B}+\alpha \Sigma \mathrm{pr}_{A}+\beta \Sigma \mathrm{pr}_{B}
$$

(4.4) Corollary. $[\alpha, \beta]_{f}=[\beta h(f)]^{-\alpha}+[-\alpha,-\beta \Sigma d]$.

Note that this corollary expresses the twisted Whitehead product in terms of the untwisted Whitehead product and the action (2.2).

As in [2] let $\pi_{1}^{B}(Z) \star \pi_{1}^{A \wedge B}(Z)$ be the semidirect product with operation

$$
(\beta, \xi) \star(\bar{\beta}, \bar{\xi})=\left(\beta+\bar{\beta}, \xi^{\bar{\beta}}+\bar{\xi}\right)
$$

Then $\pi_{1}^{A}(Z)$ acts on $\pi_{1}^{B}(Z) \star \pi_{1}^{A \wedge B}(Z)$ by $(\beta, \xi)^{\alpha}=\left(\beta,-[\alpha, \beta]+\xi^{\alpha}\right)$. From (4.4) we have:

(4.5) Proposition. If $f: A \times B \rightarrow B$ has type $\left(e, 1_{B}\right)$ then $(\beta, \beta h(f))^{\alpha}=$ $\left(\beta,-[-\alpha,-\beta]_{f}\right)$.

5. The cofiber theorem. The principal example of a twisted Whitehead product is provided by a fibration over a suspension. In this section we extend the cofiber theorem [1, Theorem 4.2] to this class of examples.

Suppose $p: E \rightarrow \Sigma X$ is a (based) Hurewicz fibration over a suspension space with fiber $F$. Let $\gamma: X \times F \rightarrow F$ be a clutching function for $p$. The class of $\gamma$ is uniquely determined by $p$ and so we write

$$
W_{p}: X * F \rightarrow \Sigma X \vee \Sigma F \quad\left(\text { rather than } W_{\gamma}\right) \text {. }
$$

We denote by $p * \varepsilon^{0}$ the fiberwise join of $p$ and the projection $\Sigma X \times S^{0} \rightarrow$ $\Sigma X$. Thus $E\left(p * \varepsilon^{0}\right)$ is the unreduced double mapping cylinder on $p$ with itself. Since spaces are well pointed we may (without changing the homotopy type) regard $E\left(p * \varepsilon^{9}\right)$ as the reduced double mapping cylinder. Doing this gives an inclusion $\Sigma X \vee \Sigma F \subset E\left(p * \varepsilon^{0}\right)$.

(5.1) THEOREM. The homotopy commutative diagram

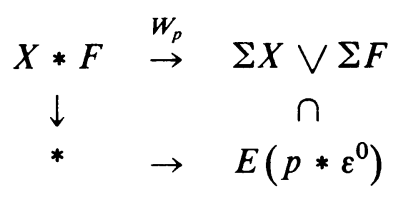

is a homotopy pushout. In particular $C_{W_{p}} \simeq E\left(p * \varepsilon^{0}\right)$. 
Proof. This follows from a direct application of Theorem 1.3 in [5].

(5.2) ExAmples. (i) For the projection fibration pr: $\Sigma A \times B \rightarrow \Sigma A$ with fiber $B, W_{\mathrm{pr}}$ is the composite

$$
A * B \stackrel{W}{\rightarrow} \Sigma A \vee \Sigma B \stackrel{r \vee r}{\rightarrow} \Sigma A \vee \Sigma B
$$

and $E\left(p * \varepsilon^{0}\right) \simeq \Sigma A \times \Sigma B$. This recovers the classical cofiber theorem $C_{W}$ $\simeq \Sigma A \times \Sigma B$ [1, Theorem 4.2].

(ii) Let $p: V_{n+1, k+1} \rightarrow S^{n}$ be the Stiefel fibration with fiber $V_{n, k}$. For $k=1$, $C_{W_{p}} \simeq S^{n} \times S^{n}$ and $W_{p} \simeq W: S^{2 n-1} \rightarrow S^{n} \vee S^{n}$. This reflects the fact that $p * \varepsilon^{0}$ is fiber homotopically trivial. Thus we obtain an alternative definition of the classical Whitehead product for spheres. However, for $k>1, W_{p} \neq$ $W: S^{n-1} * V_{n, k} \rightarrow S^{n} \vee \Sigma V_{n, k}$.

\section{REFERENCES}

1. M. Arkowitz, The generalized Whitehead product, Pacific J. Math. 12 (1962), 7-23.

2. H. J. Baues, Whitehead Produkte und Hindernisse in dem Produkt von Abbildungskegeln, Arch. Math. (Basel) 25 (1974), 184-197.

3. __ Obstruction theory: On homotopy classification of maps, Lecture Notes in Math., vol. 628, Springer, New York, 1977.

4. Peter Hilton, Homotopy theory and duality, Gordon and Breach, New York, 1965.

5. H. J. Marcum, Fibrations over double mapping cylinders (to appear).

Instituto de Matemática, Universidade Federal do Rio de Janeiro, Rio de Janeiro, BRASIL

Department of Mathematics, Sam Houston State University, Huntsville, Texas 77341

Current address: Department of Mathematics, Ohio State University, Newark Campus, Newark, Ohio 43055 